\title{
Article
}

\section{Some Extremal Graphs with Respect to Sombor Index}

\author{
Kinkar Chandra Das ${ }^{1, *} *$ and Yilun Shang ${ }^{2, *}$ (]) \\ 1 Department of Mathematics, Sungkyunkwan University, Suwon 16419, Korea \\ 2 Department of Computer and Information Sciences, Northumbria University, Newcastle NE1 8ST, UK \\ * Correspondence: kinkardas2003@gmail.com (K.C.D.); yilun.shang@northumbria.ac.uk (Y.S.)
}

check for

updates

Citation: Das, K.C.; Shang, Y. Some Extremal Graphs with Respect to Sombor Index. Mathematics 2021, 9 , 1202. https://doi.org/10.3390/ math9111202

Academic Editor: Frank Werner

Received: 18 April 2021

Accepted: 20 May 2021

Published: 25 May 2021

Publisher's Note: MDPI stays neutral with regard to jurisdictional claims in published maps and institutional affiliations.

Copyright: (c) 2021 by the authors. Licensee MDPI, Basel, Switzerland. This article is an open access article distributed under the terms and conditions of the Creative Commons Attribution (CC BY) license (https:// creativecommons.org/licenses/by/ $4.0 /)$.

\begin{abstract}
Let $G$ be a graph with set of vertices $V(G)(|V(G)|=n)$ and edge set $E(G)$. Very recently, a new degree-based molecular structure descriptor, called Sombor index is denoted by $S O(G)$ and is defined as $S O=S O(G)=\sum_{v_{i} v_{j} \in E(G)} \sqrt{d_{G}\left(v_{i}\right)^{2}+d_{G}\left(v_{j}\right)^{2}}$, where $d_{G}\left(v_{i}\right)$ is the degree of the vertex $v_{i}$ in $G$. In this paper we present some lower and upper bounds on the Sombor index of graph $G$ in terms of graph parameters (clique number, chromatic number, number of pendant vertices, etc.) and characterize the extremal graphs.
\end{abstract}

Keywords: graph; Sombor index; chromatic number; clique number

\section{Introduction}

Let $G=(V, E)$ be a graph with vertex set $V(G)=\left\{v_{1}, v_{2}, \ldots, v_{n}\right\}$ and edge set $E=E(G)$, where $|V(G)|=n$ and $|E(G)|=m$. If the vertices $v_{i}$ and $v_{j}$ are adjacent, we write $v_{i} v_{j} \in E(G)$. For $i \in\{1,2, \ldots, n\}$, let $d_{G}\left(v_{i}\right)$ be the degree of the vertex $v_{i}$. The maximum degree of a graph $G$ will be denoted by $\Delta$. A vertex $v_{i}$ of degree 1 is called a pendant vertex (also known as leaf), the edge incident with a pendant vertex is called a pendant edge. For any two nonadjacent vertices $v_{i}$ and $v_{j}$ of a graph $G$, we let $G+v_{i} v_{j}$ be the graph obtained from $G$ by adding the edge $v_{i} v_{j}$. For a subset $W$ of $V(G)$, let $G-W$ be the subgraph of $G$ obtained by deleting the vertices of $W$ and the edges incident with them. Similarly, for a subset $E^{\prime}$ of $E(G)$, we denote by $G-E^{\prime}$ the subgraph of $G$ obtained by deleting the edges of $E^{\prime}$. If $W=\left\{v_{i}\right\}$ and $E^{\prime}=\left\{v_{i} v_{j}\right\}$, the subgraphs $G-W$ and $G-E^{\prime}$ will be written as $G-v_{i}$ and $G-v_{i} v_{j}$ for short, respectively. The chromatic number of a graph $G$, denoted by $\chi(G)$, is the minimum number of colors such that vertices of $G$ can be colored with these colors in order that no two adjacent vertices have the same color. A clique of graph $G$ is a subset $V_{0}$ of $V(G)$ such that in $G\left[V_{0}\right]$, the subgraph of $G$ induced by $V_{0}$, any two vertices are adjacent. The clique number of $G$, denoted by $\omega(G)$, is the number of vertices in a largest clique of $G$. For two vertex-disjoint graphs $G_{1}$ and $G_{2}$, we denote by $G_{1} \cup G_{2}$ the graph which consists of two components $G_{1}$ and $G_{2}$. As usual, $P_{n}, C_{n}, K_{1, n-1}$ and $K_{p, q}(p+q=n)$, denote, respectively, the path, the cycle, the star and the complete bipartite graph on $n$ vertices. Other undefined notations and terminology on the graph theory can be found in [1].

A topological descriptor is a numerical descriptor of the topology of a molecule. These topological descriptors are used for predicting the physico-chemical and/or biological properties of molecules in quantitative structure-property relationship (QSPR) and quantitative structure-activity relationship (QSAR) studies $[2,3]$. In the literature, several degree- and distance-based topological descriptors were proposed and studied by some researchers [4-17]. Very recently, a new degree-based molecular structure descriptor was introduced, the Sombor index is denoted by $S O(G)$ and is defined as follows [18]:

$$
S O=S O(G)=\sum_{v_{i} v_{j} \in E(G)} \sqrt{d_{G}\left(v_{i}\right)^{2}+d_{G}\left(v_{j}\right)^{2}} .
$$


Many fundamental mathematical properties such as lower and upper bounds can be found in, e.g., $[3,10,18-26]$. This topological index was motivated by the geometric interpretation of the degree radius of an edge $v_{i} v_{j}$, which is the distance from the origin to the ordered pair $\left(d_{G}\left(v_{i}\right), d_{G}\left(v_{j}\right)\right)$.

Denote by $\mathcal{W}(n, \omega)$ the set of connected graphs of order $n$ with clique number $\omega$. The long kite graph $K i_{n, \omega}$ (see, Figure 1 ) is a graph of order $n$ obtained from a clique $K_{\omega}$ and a path $P_{n-\omega}$ by adding an edge between a vertex from the clique and an endpoint from the path. In particular, for $\omega=n, K i_{n, \omega} \cong K_{n}$. Let $G \cong K i_{n, \omega}$. For $\omega \leq n-2$, we have

$$
\begin{aligned}
& \sum_{\substack{v_{i} v_{j} \in E(G) \\
v_{i}, v_{j} \in V(K \omega)}} \sqrt{d_{G}\left(v_{i}\right)^{2}+d_{G}\left(v_{j}\right)^{2}} \\
= & \left(\begin{array}{c}
\omega-1 \\
2
\end{array}\right) \sqrt{(\omega-1)^{2}+(\omega-1)^{2}}+(\omega-1) \sqrt{\omega^{2}+(\omega-1)^{2}} \\
= & \left(\begin{array}{c}
\omega-1 \\
2
\end{array}\right) \sqrt{2}(\omega-1)+(\omega-1) \sqrt{2 \omega^{2}-2 \omega+1}, \\
& \sum_{\substack{v_{i} v_{j} \in E(G) \\
v_{i}, v_{j} \in V(G-V(K \omega))}} \sqrt{d_{G}\left(v_{i}\right)^{2}+d_{G}\left(v_{j}\right)^{2}}=(n-\omega-2) \sqrt{8}+\sqrt{5}
\end{aligned}
$$

and hence

$$
\begin{gathered}
S O\left(K i_{n, \omega}\right)=\sum_{\substack{v_{i} v_{j} \in E(G) \\
v_{i}, v_{j} \in V\left(K_{\omega}\right)}} \sqrt{d_{G}\left(v_{i}\right)^{2}+d_{G}\left(v_{j}\right)^{2}}+\sum_{\substack{v_{i} v_{j} \in E(G) \\
v_{i}, v_{j} \in V(G-V(K \omega))}} \sqrt{d_{G}\left(v_{i}\right)^{2}+d_{G}\left(v_{j}\right)^{2}} \\
+\sqrt{\omega^{2}+4} \\
=\left(\begin{array}{c}
\omega-1 \\
2
\end{array}\right) \sqrt{2}(\omega-1)+(\omega-1) \sqrt{2 \omega^{2}-2 \omega+1}+\sqrt{\omega^{2}+4} \\
+(n-\omega-2) \sqrt{8}+\sqrt{5} .
\end{gathered}
$$

In this paper, we present a lower bound on $S O$ of graph $G$ in terms of $n$ and clique number $\omega$, and characterize the extremal graphs.

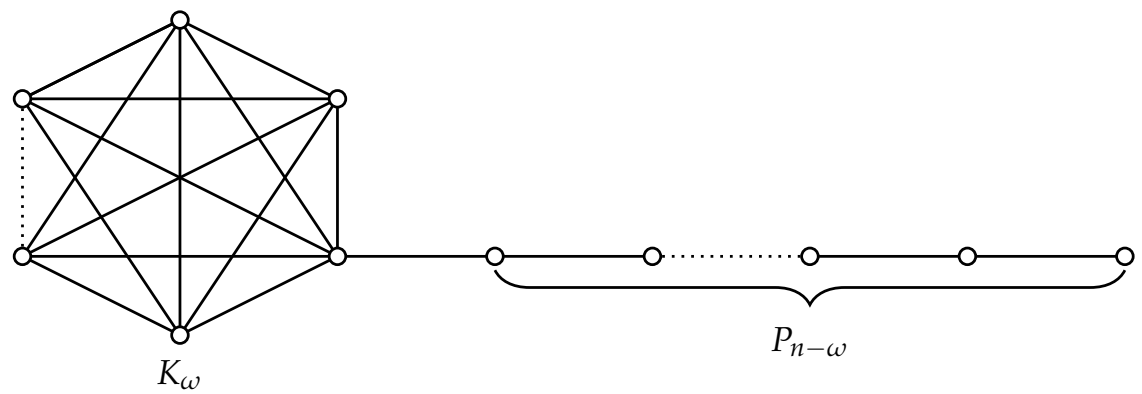

$$
K i_{n, \omega}
$$

Figure 1. The long kite graph $K i_{n, \omega}$.

Theorem 1. Let $G \in \mathcal{W}(n, \omega)$. Then $S O(G) \geq S O\left(K i_{n, \omega}\right)$ with equality holding if and only if $G \cong K i_{n, \omega}$.

Corollary 1. [18] Let $G$ be a connected graph of order $n$. Then $S O(G) \geq S O\left(P_{n}\right)$ with equality holding if and only if $G \cong P_{n}$. 
Proof of Corollary 1. Let $\omega$ be the clique number of graph $G$. Then $\omega \geq 2$. Therefore one can easily see that

$$
S O(G) \geq S O\left(K i_{n, \omega}\right) \geq S O\left(K i_{n, \omega-1}\right) \geq \cdots \geq S O\left(K i_{n, 3}\right) \geq S O\left(K i_{n, 2}\right)=S O\left(P_{n}\right) .
$$

hence we obtain the required result.

Let $\mathcal{X}(n, k)$ be the set of connected graphs of order $n$ with chromatic number $k$. Recall that the Turán graph $T_{n}(k)$ is a complete $k$-partite graph of order $n$ whose partition sets differ in size by at most 1 . When $k=n$, the only graph in $\mathcal{X}(n, k)$ is $K_{n}$. So, we now assume that $n=k q+r$ where $0 \leq r<k$, i.e., $q=\left\lfloor\frac{n}{k}\right\rfloor$ in $\mathcal{X}(n, k)$. We now give an upper bound on $S O$ of graph $G$ in terms of $n$ and chromatic number $k$, and characterize the extremal graphs.

Theorem 2. For any graph $G \in \mathcal{X}(n, k)$, we have

$$
\begin{aligned}
S O(G) \leq & r(k-r)\left\lfloor\frac{n}{k}\right\rfloor\left\lceil\frac{n}{k}\right\rceil \sqrt{\left(n-\left\lfloor\frac{n}{k}\right\rfloor\right)^{2}+\left(n-\left\lceil\frac{n}{k}\right\rceil\right)^{2}}+\sqrt{2}\left(\begin{array}{l}
r \\
2
\end{array}\right)\left\lceil\frac{n}{k}\right\rceil^{2}\left(n-\left\lceil\frac{n}{k}\right\rceil\right) \\
& +\sqrt{2}\left(\begin{array}{c}
k-r \\
2
\end{array}\right)\left\lfloor\frac{n}{k}\right\rfloor^{2}\left(n-\left\lfloor\frac{n}{k}\right\rfloor\right)
\end{aligned}
$$

with equality holding if and only if $G \cong T_{n}(k)$.

Recall that a short kite graph $K i_{n}^{\omega}$ obtained by adding $n-\omega$ pendant vertices to the unique vertex of clique $K_{\omega}$; see Figure 2 . Let $G \cong K i_{n}^{n-p}$. We have

$$
\begin{gathered}
\sum_{\substack{v_{i} v_{j} \in E(G) \\
v_{i}, v_{j} \in V\left(K_{\omega}\right)}} \sqrt{d_{G}\left(v_{i}\right)^{2}+d_{G}\left(v_{j}\right)^{2}}=\frac{(n-p-1)(n-p-2)}{2} \sqrt{(n-p-1)^{2}+(n-p-1)^{2}} \\
+(n-p-1) \sqrt{(n-1)^{2}+(n-p-1)^{2}} \\
=\frac{(n-p-1)(n-p-2)}{\sqrt{2}}(n-p-1) \\
+(n-p-1) \sqrt{(n-1)^{2}+(n-p-1)^{2}}
\end{gathered}
$$

and hence

$$
\begin{gathered}
S O\left(K i_{n}^{n-p}\right)=\sum_{\substack{v_{i} v_{j} \in E(G) \\
v_{i}, v_{j} \in V\left(K_{\omega}\right)}} \sqrt{d_{G}\left(v_{i}\right)^{2}+d_{G}\left(v_{j}\right)^{2}}+\sum_{\substack{v_{i} v_{j} \in E(G), v_{i} \in V\left(K_{\omega}\right) \\
v_{j} \in V\left(G-V\left(K_{\omega}\right)\right)}} \sqrt{d_{G}\left(v_{i}\right)^{2}+d_{G}\left(v_{j}\right)^{2}} \\
=p \sqrt{(n-1)^{2}+1}+\frac{(n-p-1)(n-p-2)}{\sqrt{2}}(n-p-1) \\
+(n-p-1) \sqrt{(n-1)^{2}+(n-p-1)^{2}} .
\end{gathered}
$$

Finally we give an upper bound on Sombor index in terms of $n, p$ pendant vertices, and characterize the extremal graphs. 


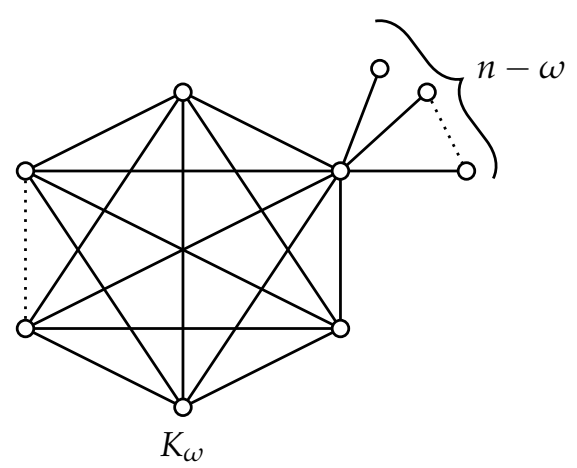

$K i_{n}^{\omega}$

Figure 2. The short kite graph $K i_{n}^{\omega}$.

Theorem 3. Let $G$ be a graph of order $n$ with $p$ pendant vertices. Then

$$
S O(G) \leq S O\left(K i_{n}^{n-p}\right)
$$

with equality holding if and only if $G \cong K i_{n}^{n-p}$.

\section{Preliminaries}

From the definition of Sombor index, we have

Lemma 1. Let $G$ be a graph. Then $S O(G)>S O(G-e)$, where e is any edge in $G$.

Lemma 2. [18] Let $T$ be a tree of order $n$. Then $S O(T) \geq(n-3) \sqrt{8}+2 \sqrt{5}$ with equality if and only if $T \cong P_{n}$.

Lemma 3. [10] Let $T$ be a tree of order $n(>4)$ with $T ¥ P_{n}$. Then $S O(T)>(n-1) \sqrt{8}$.

In [10], the following two sets are defined:

$$
\begin{aligned}
& A=\left\{v_{i} v_{j} \in E(G): d_{G}\left(v_{i}\right)=2>1=d_{G}\left(v_{j}\right)\right\} \\
& D=\left\{v_{i} v_{j} \in E(G): d_{G}\left(v_{i}\right) \geq 3, d_{G}\left(v_{j}\right) \geq 2 \text { or } d_{G}\left(v_{i}\right) \geq 4, d_{G}\left(v_{j}\right)=1\right\} .
\end{aligned}
$$

The following result has been proved in [10].

Lemma 4. [10] Let $T\left(¥ P_{n}\right)$ be a tree of order $n$. Then $|D| \geq|A|$.

\section{Proofs}

Proof of Theorem 1. For $\omega=n$, we have $G \cong K_{n}$ and hence the equality holds. For $\omega=n-1, K i_{n, n-1}$ is a subgraph of $G$ as $G \in \mathcal{W}(n, \omega)$. Hence by Lemma 1 , we obtain

$$
S O(G) \geq S O\left(K i_{n, n-1}\right)=\left(\begin{array}{c}
n-2 \\
2
\end{array}\right) \sqrt{2}(n-2)+(n-2) \sqrt{2 n^{2}-6 n+5}+\sqrt{n^{2}-2 n+2}
$$

with equality if and only if $G \cong K i_{n, n-1}$. For $\omega=2$, we have $G \cong T_{n}$ or $T_{n}$ is a subgraph of $G$, where $T_{n}$ is a tree of order $n$. By Lemmas 1 and 2, we have $S O(G) \geq S O\left(T_{n}\right) \geq$ $(n-3) \sqrt{8}+2 \sqrt{5}$. Moreover, $S O(G)=(n-3) \sqrt{8}+2 \sqrt{5}$ if and only if $G \cong P_{n}$.

Otherwise, $3 \leq \omega \leq n-2$. Since the clique number of $G$ is $\omega$, we can assume that a clique of $G$ is $S(G)=\left\{v_{1}, v_{2}, \ldots, v_{\omega}\right\}$. Let $H$ be a connected graph with $H \subseteq G$ such that $V(H)=V(G)$ and $H-E\left(K_{\omega}\right) \cong T_{1} \cup T_{2} \cup \cdots \cup T_{\omega}$, where $T_{1}, T_{2}, \ldots, T_{\omega}$ are the trees with $v_{i} \in V\left(T_{i}\right),\left|V\left(T_{i}\right)\right|=n_{i}(1 \leq i \leq \omega), n_{1} \geq n_{2} \geq \cdots \geq n_{\omega}$ and $n_{1}+n_{2}+\cdots+n_{\omega}=n$. Moreover, $S(G)=S(H)$. By Lemma 1 , we have $S O(G) \geq S O(H)$ with equality if and only 
if $G \cong H$. For $1 \leq i \leq \omega$, we have $v_{i} \in V\left(T_{i}\right)$ and $d_{H}\left(v_{i}\right)=d_{T_{i}}\left(v_{i}\right)+\omega-1$. Moreover, $d_{H}\left(v_{i}\right)=d_{T}\left(v_{i}\right)$ for $\omega+1 \leq i \leq n$ and $T \in\left\{T_{1}, \ldots, T_{\omega}\right\}$. Thus, we have

$$
\begin{aligned}
S O(G) & =\sum_{v_{i} v_{j} \in E(G)} \sqrt{d_{G}\left(v_{i}\right)^{2}+d_{G}\left(v_{j}\right)^{2}} \\
& \geq \sum_{v_{i} v_{j} \in E(H)} \sqrt{d_{H}\left(v_{i}\right)^{2}+d_{H}\left(v_{j}\right)^{2}} \\
& =\sum_{v_{i} v_{j} \in E\left(K_{\omega}\right)} \sqrt{d_{H}\left(v_{i}\right)^{2}+d_{H}\left(v_{j}\right)^{2}}+\sum_{\substack{v_{i} v_{j} \in E(H), v_{i} \in V\left(G-V\left(K_{\omega}\right)\right), v_{j} \in\left(K_{\omega}\right) \text { Or } v_{j} \in V\left(G-W\left(K_{\omega}\right)\right)}} \sqrt{d_{H}\left(v_{i}\right)^{2}+d_{H}\left(v_{j}\right)^{2}} \\
& =\sum_{v_{i} v_{j} \in E\left(K_{\omega}\right)} \sqrt{d_{H}\left(v_{i}\right)^{2}+d_{H}\left(v_{j}\right)^{2}}+\sum_{k=1}^{\omega} \sum_{v_{i} v_{j} \in E\left(T_{k}\right)} \sqrt{d_{H}\left(v_{i}\right)^{2}+d_{H}\left(v_{j}\right)^{2}} .
\end{aligned}
$$

Claim 1. For $2 \leq i \leq \omega, \sum_{v_{k} v_{\ell} \in E\left(T_{i}\right)} \sqrt{d_{H}\left(v_{k}\right)^{2}+d_{H}\left(v_{\ell}\right)^{2}} \geq\left(n_{i}-1\right) \sqrt{8}$ with equality if and only if $n_{i}=1$.

Proof of Claim 1. Let

$$
A_{i}=\sum_{v_{k} v_{\ell} \in E\left(T_{i}\right)} \sqrt{d_{H}\left(v_{k}\right)^{2}+d_{H}\left(v_{\ell}\right)^{2}}, \quad 2 \leq i \leq \omega .
$$

For $n_{i}=1(2 \leq i \leq \omega)$, then $A_{i}=\left(n_{i}-1\right) \sqrt{8}=0$, the equality holds in Claim 1. For $n_{i}=2(2 \leq i \leq \omega)$, then

$$
A_{i}=\sqrt{\omega^{2}+1}>\left(n_{i}-1\right) \sqrt{8}
$$

as $\omega \geq 3$, the inequality strictly holds in Claim 1 . Otherwise, $n_{i} \geq 3$. First we assume that $T_{i} \cong P_{n_{i}}$. Thus, we have $d_{T_{i}}\left(v_{i}\right)=1$ or $d_{T_{i}}\left(v_{i}\right)=2$. When $d_{T_{i}}\left(v_{i}\right)=1$, we obtain

$$
A_{i}=\sqrt{\omega^{2}+4}+\left(n_{i}-3\right) \sqrt{8}+\sqrt{5}>\left(n_{i}-1\right) \sqrt{8}
$$

as $\omega \geq 3$ and $\sqrt{13}+\sqrt{5}>2 \sqrt{8}$. When $d_{T_{i}}\left(v_{i}\right)=2$, we obtain

$$
\begin{aligned}
A_{i} & =\sqrt{\omega^{2}+4}+\sqrt{\omega^{2}+1}+\left(n_{i}-4\right) \sqrt{8}+\sqrt{5}>\left(n_{i}-1\right) \sqrt{8} \\
\text { or } A_{i} & =2 \sqrt{\omega^{2}+4}+\left(n_{i}-5\right) \sqrt{8}+2 \sqrt{5}>\left(n_{i}-1\right) \sqrt{8}
\end{aligned}
$$

as $\omega \geq 3$ and $\sqrt{13}+\sqrt{5}>2 \sqrt{8}$.

Next we assume that $T_{i} \nsucceq P_{n_{i}}$. Since $d_{H}\left(v_{i}\right)>d_{T_{i}}\left(v_{i}\right)(2 \leq i \leq \omega)$ with Lemma 3 , we obtain

$$
A_{i}>\sum_{v_{k} v_{\ell} \in E\left(T_{i}\right)} \sqrt{d_{T_{i}}\left(v_{k}\right)^{2}+d_{T_{i}}\left(v_{\ell}\right)^{2}}>\left(n_{i}-1\right) \sqrt{8} .
$$

Claim 1 is proved.

Claim 2.

$$
\sum_{v_{k} v_{\ell} \in E\left(T_{1}\right)} \sqrt{d_{H}\left(v_{k}\right)^{2}+d_{H}\left(v_{\ell}\right)^{2}} \geq \begin{cases}\sqrt{\omega^{2}+1} & \text { if } n_{1}=2, \\ \sqrt{\omega^{2}+4}+\left(n_{1}-3\right) \sqrt{8}+\sqrt{5} & \text { if } n_{1} \geq 3\end{cases}
$$

with equality if and only if $T_{1} \cong P_{n_{1}}$ with $d_{T_{1}}\left(v_{1}\right)=1$.

Proof of Claim 2. Let

$$
A_{1}=\sum_{v_{k} v_{\ell} \in E\left(T_{1}\right)} \sqrt{d_{H}\left(v_{k}\right)^{2}+d_{H}\left(v_{\ell}\right)^{2}} .
$$


Since $\omega \leq n-2$ and $n_{1} \geq n_{i}(2 \leq i \leq \omega)$, we have $n_{1} \geq 2$. For $n_{1}=2$, we have $A_{1}=\sqrt{\omega^{2}+1}$, the equality holds in Claim 2. Otherwise, $n_{1} \geq 3$. We have $v_{1} \in V\left(T_{1}\right)$. First we assume that $T_{1} \cong P_{n_{1}}$. Thus, we have $d_{T_{1}}\left(v_{1}\right)=1$ or $d_{T_{1}}\left(v_{1}\right)=2$. If $d_{T_{1}}\left(v_{1}\right)=1$, then we obtain

$$
A_{1}=\sqrt{\omega^{2}+4}+\left(n_{1}-3\right) \sqrt{8}+\sqrt{5} .
$$

The equality holds in Claim 2. Otherwise, $d_{T_{1}}\left(v_{1}\right)=2$. We consider two cases:

Case 1. $n_{1}=3$. In this case

$$
A_{1}=2 \sqrt{(\omega+1)^{2}+1}>\sqrt{\omega^{2}+4}+\left(n_{1}-3\right) \sqrt{8}+\sqrt{5}
$$

as $\omega \geq 3$.

Case 2. $n_{1} \geq 4$. We obtain

$$
\begin{aligned}
& A_{1}=\sqrt{(\omega+1)^{2}+4}+\sqrt{(\omega+1)^{2}+1}+\left(n_{1}-4\right) \sqrt{8}+\sqrt{5} \\
& \quad>\sqrt{\omega^{2}+4}+\left(n_{1}-3\right) \sqrt{8}+\sqrt{5} \\
& \text { or } A_{1}=2 \sqrt{(\omega+1)^{2}+4}+\left(n_{1}-5\right) \sqrt{8}+2 \sqrt{5}>\sqrt{\omega^{2}+4}+\left(n_{1}-3\right) \sqrt{8}+\sqrt{5}
\end{aligned}
$$

as $\omega \geq 3$ and $\sqrt{20}+\sqrt{5}>2 \sqrt{8}$.

Next we assume that $T_{1} \nRightarrow P_{n_{1}}$. Then $n_{1} \geq 4$. If $n_{1}=4$, then $T_{1} \cong K_{1,3}$ and one can easily check that $A_{1}>\sqrt{\omega^{2}+4}+\left(n_{1}-3\right) \sqrt{8}+\sqrt{5}$. Otherwise, $n_{1} \geq 5$. We consider the following two cases:

Case 3. $d_{T_{1}}\left(v_{1}\right)=1$. Let $v_{r}$ be a vertex adjacent to $v_{1}$ in tree $T_{1}$. Then $d_{T_{1}}\left(v_{r}\right) \geq 2$ as $n_{1} \geq 5$. First suppose that $T_{1}-v_{1} \cong P_{n_{1}-1}$. Then $d_{T_{1}}\left(v_{r}\right)=3$. One can easily see that

$$
\begin{aligned}
A_{1} & =\sqrt{\left(d_{T_{1}}\left(v_{1}\right)+\omega-1\right)^{2}+9}+\sum_{v_{k} v_{\ell} \in E\left(T_{1}-v_{1}\right)} \sqrt{d_{H}\left(v_{k}\right)^{2}+d_{H}\left(v_{\ell}\right)^{2}} \\
& =\sqrt{\omega^{2}+9}+\sum_{v_{k} v_{\ell} \in E\left(T_{1}-v_{1}\right)} \sqrt{d_{T_{1}}\left(v_{k}\right)^{2}+d_{T_{1}}\left(v_{\ell}\right)^{2}} \\
& >\sqrt{\omega^{2}+9}+\sqrt{13}+\left(n_{1}-4\right) \sqrt{8}+\sqrt{5}>\sqrt{\omega^{2}+4}+\left(n_{1}-3\right) \sqrt{8}+\sqrt{5}
\end{aligned}
$$

as $\sqrt{13}+\sqrt{5}>2 \sqrt{8}$.

Next suppose that $T_{1}-v_{1} \nsucceq P_{n_{1}-1}$. In this case $d_{T_{1}}\left(v_{r}\right) \geq 2$. Since $T_{1}-v_{1}$ is a tree of order $n_{1}-1$, by Lemma 3 , we obtain

$$
\begin{aligned}
A_{1} & =\sqrt{\left(d_{T_{1}}\left(v_{1}\right)+\omega-1\right)^{2}+d_{T_{1}}\left(v_{r}\right)^{2}}+\sum_{v_{k} v_{\ell} \in E\left(T_{1}-v_{1}\right)} \sqrt{d_{H}\left(v_{k}\right)^{2}+d_{H}\left(v_{\ell}\right)^{2}} \\
& \geq \sqrt{\omega^{2}+4}+\sum_{v_{k} v_{\ell} \in E\left(T_{1}-v_{1}\right)} \sqrt{d_{T_{1}}\left(v_{k}\right)^{2}+d_{T_{1}}\left(v_{\ell}\right)^{2}} \\
& >\sqrt{\omega^{2}+4}+\sum_{v_{k} v_{\ell} \in E\left(T_{1}-v_{1}\right)} \sqrt{d_{T_{1}-v_{1}}\left(v_{k}\right)^{2}+d_{T_{1}-v_{1}}\left(v_{\ell}\right)^{2}} \\
& >\sqrt{\omega^{2}+4}+\left(n_{1}-2\right) \sqrt{8}>\sqrt{\omega^{2}+4}+\left(n_{1}-3\right) \sqrt{8}+\sqrt{5} .
\end{aligned}
$$

Case 4. $d_{T_{1}}\left(v_{1}\right) \geq 2$. For $d_{T_{1}}\left(v_{1}\right)=n_{1}-1$, one can easily check that $A_{1}>\sqrt{\omega^{2}+4}+$ $\left(n_{1}-3\right) \sqrt{8}+\sqrt{5}$. So now we have $d_{T_{1}}\left(v_{1}\right) \leq n_{1}-2$. Since $n_{1} \geq 5$ and $T_{1}$ is a tree, $T_{1}-v_{1}=\ell K_{1} \cup T_{1}^{1} \cup T_{1}^{2} \cup \cdots \cup T_{1}^{k}(\ell \geq 0, k \geq 1)$, (say), where $T_{1}^{i}(1 \leq i \leq k)$ is a tree of order $n_{1}^{i}\left(=\left|V\left(T_{1}^{i}\right)\right| \geq 2\right)$ with $\sum_{i=1}^{k} n_{1}^{i}=n_{1}-\ell-1$. Thus, we have $d_{H}\left(v_{1}\right)=$ 
$d_{T_{1}}\left(v_{1}\right)+\omega-1=\omega+k+\ell-1$. Let $v_{r}$ be a vertex in $T_{1}^{i}$ such that $v_{1} v_{r} \in E\left(T_{1}\right)$, where $1 \leq i \leq k$. We now prove that

$$
\sum_{v_{j} v_{\ell} \in E\left(T_{1}^{i}\right)} \sqrt{d_{T_{1}}\left(v_{j}\right)^{2}+d_{T_{1}}\left(v_{\ell}\right)^{2}} \geq\left(n_{1}^{i}-2\right) \sqrt{8}+\sqrt{5} .
$$

First we assume that $T_{1}^{i} \supsetneqq P_{n_{1}^{i}}$. Then by Lemma $3, S O\left(T_{1}^{\prime}\right)>\left(n_{1}^{i}-1\right) \sqrt{8}$. We obtain

$$
\begin{aligned}
\sum_{v_{j} v_{\ell} \in E\left(T_{1}^{i}\right)} \sqrt{d_{T_{1}}\left(v_{j}\right)^{2}+d_{T_{1}}\left(v_{\ell}\right)^{2}} & >\sum_{v_{j} v_{\ell} \in E\left(T_{1}^{i}\right)} \sqrt{d_{T_{1}^{i}}\left(v_{j}\right)^{2}+d_{T_{1}^{i}}\left(v_{\ell}\right)^{2}} \\
& =S O\left(T_{1}^{i}\right) \\
& >\left(n_{1}^{i}-1\right) \sqrt{8}>\left(n_{1}^{i}-2\right) \sqrt{8}+\sqrt{5}
\end{aligned}
$$

the result strictly holds in (3).

Next we assume that $T_{1}^{i} \cong P_{n_{1}^{i}}$. For $d_{T_{1}^{i}}\left(v_{r}\right)=1$, we have $d_{T_{1}}\left(v_{r}\right)=d_{T_{1}^{i}}\left(v_{r}\right)+1=2$, $d_{T_{1}}\left(v_{k}\right)=d_{T_{1}^{i}}\left(v_{k}\right)$ for $v_{k} \in V\left(T_{1}^{i}-v_{r}\right)$, and hence we obtain

$$
\sum_{v_{j} v_{\ell} \in E\left(T_{1}^{i}\right)} \sqrt{d_{T_{1}}\left(v_{j}\right)^{2}+d_{T_{1}}\left(v_{\ell}\right)^{2}}=\left(n_{1}^{i}-2\right) \sqrt{8}+\sqrt{5} .
$$

The equality holds in (3). Otherwise, $d_{T_{1}^{i}}\left(v_{r}\right)=2$. In this case $d_{T_{1}}\left(v_{r}\right)=d_{T_{1}^{i}}\left(v_{r}\right)+1=$ 3 and $d_{T_{1}}\left(v_{k}\right)=d_{T_{1}^{i}}\left(v_{k}\right)$ for $v_{k} \in V\left(T_{1}^{i}-v_{r}\right)$. If $2 \leq n_{1}^{i} \leq 3$, then one can easily check that the result holds in (3). Otherwise, $n_{1}^{i} \geq 4$. We obtain

$$
\sum_{v_{j} v_{\ell} \in E\left(T_{1}^{i}\right)} \sqrt{d_{T_{1}}\left(v_{j}\right)^{2}+d_{T_{1}}\left(v_{\ell}\right)^{2}}=2 \sqrt{13}+2 \sqrt{5}+\left(n_{1}^{i}-5\right) \sqrt{8}
$$

or

$$
\sum_{v_{j} v_{\ell} \in E\left(T_{1}^{i}\right)} \sqrt{d_{T_{1}}\left(v_{j}\right)^{2}+d_{T_{1}}\left(v_{\ell}\right)^{2}}=\sqrt{13}+\sqrt{10}+2 \sqrt{5}+\left(n_{1}^{i}-5\right) \sqrt{8} .
$$

One can easily check that

$$
\sum_{v_{j} v_{\ell} \in E\left(T_{1}^{i}\right)} \sqrt{d_{T_{1}}\left(v_{j}\right)^{2}+d_{T_{1}}\left(v_{\ell}\right)^{2}}>\left(n_{1}^{i}-2\right) \sqrt{8}+\sqrt{5} \text { as } \sqrt{13}+\sqrt{5}>2 \sqrt{8} .
$$

Again the result holds in (3).

Using the result in (3), we obtain

$$
\begin{aligned}
A_{1}= & \sum_{v_{k} v_{\ell} \in E\left(T_{1}\right)} \sqrt{d_{H}\left(v_{k}\right)^{2}+d_{H}\left(v_{\ell}\right)^{2}} \\
= & \sum_{v_{i}: v_{1} v_{i} \in E\left(T_{1}\right)} \sqrt{d_{H}\left(v_{1}\right)^{2}+d_{H}\left(v_{i}\right)^{2}}+\sum_{i=1}^{k} \sum_{v_{j} v_{\ell} \in E\left(T_{1}^{i}\right)} \sqrt{d_{H}\left(v_{j}\right)^{2}+d_{H}\left(v_{\ell}\right)^{2}} \\
= & \ell \sqrt{d_{H}\left(v_{1}\right)^{2}+1}+\sum_{\substack{v_{i}: v_{1} v_{i} \in E\left(T_{1}\right) \\
d_{T_{1}}\left(v_{i}\right) \geq 2}} \sqrt{d_{H}\left(v_{1}\right)^{2}+d_{H}\left(v_{i}\right)^{2}} \\
& \quad+\sum_{i=1}^{k} \sum_{v_{j} v_{\ell} \in E\left(T_{1}^{i}\right)} \sqrt{d_{T_{1}}\left(v_{j}\right)^{2}+d_{T_{1}}\left(v_{\ell}\right)^{2}} \\
\geq & \ell \sqrt{d_{H}\left(v_{1}\right)^{2}+1}+k \sqrt{d_{H}\left(v_{1}\right)^{2}+4}+\sum_{i=1}^{k}\left[\left(n_{1}^{i}-2\right) \sqrt{8}+\sqrt{5}\right] \\
= & \ell \sqrt{(\omega+k+\ell-1)^{2}+1}+k \sqrt{(\omega+k+\ell-1)^{2}+4} \\
& +\left(n_{1}-1-\ell-2 k\right) \sqrt{8}+k \sqrt{5} .
\end{aligned}
$$


If $\ell \geq 1$, then from (5), we obtain

$$
\begin{aligned}
& A_{1}>\sqrt{\omega^{2}+4}+(\ell-1) \sqrt{(\omega+k+\ell-1)^{2}+1}+k \sqrt{(\omega+k+\ell-1)^{2}+4} \\
&+\left(n_{1}-1-\ell-2 k\right) \sqrt{8}+k \sqrt{5} \\
& \geq \sqrt{\omega^{2}+4}+\sqrt{5}+\left(n_{1}-1-2 k\right) \sqrt{8}+(k-1)(\sqrt{13}+\sqrt{5}) \\
&>\sqrt{\omega^{2}+4}+\sqrt{5}+\left(n_{1}-3\right) \sqrt{8}
\end{aligned}
$$

as $\sqrt{13}+\sqrt{5}>2 \sqrt{8}$. Otherwise, $\ell=0$. In this case $k \geq 2$. From (5), we obtain

$$
\begin{aligned}
A_{1} & \geq k \sqrt{(\omega+k-1)^{2}+4}+\left(n_{1}-1-2 k\right) \sqrt{8}+k \sqrt{5} \\
& >\sqrt{\omega^{2}+4}+\sqrt{5}+\left(n_{1}-1-2 k\right) \sqrt{8}+(k-1)(\sqrt{13}+\sqrt{5}) \\
& >\sqrt{\omega^{2}+4}+\sqrt{5}+\left(n_{1}-3\right) \sqrt{8}
\end{aligned}
$$

as $\sqrt{13}+\sqrt{5}>2 \sqrt{8}$. Claim 2 is proved.

Using Claim 1 and Claim 2, we obtain

$$
\sum_{k=1}^{\omega} \sum_{v_{i} v_{j} \in E\left(T_{k}\right)} \sqrt{d_{H}\left(v_{i}\right)^{2}+d_{H}\left(v_{j}\right)^{2}} \geq \sqrt{\omega^{2}+4}+(n-\omega-2) \sqrt{8}+\sqrt{5}
$$

with equality if and only if $n_{2}=\cdots=n_{\omega}=1$ and $T_{1} \cong P_{n_{1}}$ with $d_{T_{1}}\left(v_{1}\right)=1$, i.e., $G \cong K i_{n, \omega}$.

Since $n \geq \omega+2$, we have $d_{H}\left(v_{1}\right) \geq \omega$ and $d_{H}\left(v_{i}\right) \geq \omega-1(2 \leq i \leq \omega)$. Using the above result in (3), we obtain

$$
\begin{aligned}
S O(G) \geq & \sum_{i=2}^{\omega} \sqrt{d_{H}\left(v_{1}\right)^{2}+d_{H}\left(v_{i}\right)^{2}}+\sum_{\substack{v_{i} v_{j} \in E(K \omega) \\
2 \leq i<j \leq \omega}} \sqrt{d_{H}\left(v_{i}\right)^{2}+d_{H}\left(v_{j}\right)^{2}} \\
& +\sum_{k=1}^{\omega} \sum_{v_{i} v_{j} \in E\left(T_{k}\right)} \sqrt{d_{H}\left(v_{i}\right)^{2}+d_{H}\left(v_{j}\right)^{2}} \\
& \geq(\omega-1) \sqrt{2 \omega^{2}-2 \omega+1}+\left(\begin{array}{c}
\omega-1 \\
2
\end{array}\right) \sqrt{2}(\omega-1)+\sqrt{\omega^{2}+4} \\
& +(n-\omega-2) \sqrt{8}+\sqrt{5} \\
& =S O\left(K i_{n, \omega}\right) .
\end{aligned}
$$

This completes the proof of the theorem.

Let $n_{1}, n_{2}, \ldots, n_{k}$ be positive integers with $\sum_{i=1}^{k} n_{i}=n$. Denote by $K_{n_{1}, n_{2}, \ldots, n_{k}}$ a complete $k$-partite graph of order $n$ whose partition sets are of size $n_{1}, n_{2}, \ldots, n_{k}$, respectively. We will determine the extremal graph in $\mathcal{X}(n, k)$ with respect to Sombor index of graphs $G$. For this we first prove a related lemma below.

Lemma 5. Let $K_{n_{1}, n_{2}, \ldots, n_{k}}$ be a graph defined as above with $n_{i}-n_{j} \geq 2$ for $i<j$. Then

$$
S O\left(K_{n_{1}, n_{2}, \ldots, n_{i}, \ldots, n_{j}, \ldots, n_{k}}\right)<S O\left(K_{n_{1}, n_{2}, \ldots, n_{i}-1, \ldots, n_{j}+1, \ldots, n_{k}}\right) .
$$


Proof. Without loss of generality, we can assume that $n_{1}-n_{2} \geq 2$. This lemma will be proved if we can prove the following:

$$
S O\left(K_{n_{1}, n_{2}, \ldots, n_{k}}\right)<S O\left(K_{n_{1}-1, n_{2}+1, \ldots, n_{k}}\right) .
$$

By the definition of Sombor index, we obtain

$$
\begin{aligned}
S O\left(K_{n_{1}, n_{2}, \ldots, n_{k}}\right)= & \sum_{1 \leq i<j \leq k} n_{i} n_{j} \sqrt{\left(n-n_{i}\right)^{2}+\left(n-n_{j}\right)^{2}} \\
= & \sum_{3 \leq i<j \leq k} n_{i} n_{j} \sqrt{\left(n-n_{i}\right)^{2}+\left(n-n_{j}\right)^{2}}+n_{1} n_{2} \sqrt{\left(n-n_{1}\right)^{2}+\left(n-n_{2}\right)^{2}} \\
& +n_{1} \sum_{i=3}^{k} n_{i} \sqrt{\left(n-n_{1}\right)^{2}+\left(n-n_{i}\right)^{2}}+n_{2} \sum_{i=3}^{k} n_{i} \sqrt{\left(n-n_{2}\right)^{2}+\left(n-n_{i}\right)^{2}} .
\end{aligned}
$$

Then, in view of the fact that $n_{1}-1 \geq n_{2}+1$, we obtain

$$
\begin{aligned}
& S O\left(K_{n_{1}-1, n_{2}+1, n_{3}, \ldots, n_{k}}\right)-S O\left(K_{n_{1}, n_{2}, n_{3}, \ldots, n_{k}}\right) \\
& =\left(n_{1}-1\right)\left(n_{2}+1\right) \sqrt{\left(n-n_{1}+1\right)^{2}+\left(n-n_{2}-1\right)^{2}}-n_{1} n_{2} \sqrt{\left(n-n_{1}\right)^{2}+\left(n-n_{2}\right)^{2}} \\
& +\left(n_{1}-1\right) \sum_{i=3}^{k} n_{i} \sqrt{\left(n-n_{1}+1\right)^{2}+\left(n-n_{i}\right)^{2}}-n_{1} \sum_{i=3}^{k} n_{i} \sqrt{\left(n-n_{1}\right)^{2}+\left(n-n_{i}\right)^{2}} \\
& +\left(n_{2}+1\right) \sum_{i=3}^{k} n_{i} \sqrt{\left(n-n_{2}-1\right)^{2}+\left(n-n_{i}\right)^{2}}-n_{2} \sum_{i=3}^{k} n_{i} \sqrt{\left(n-n_{2}\right)^{2}+\left(n-n_{i}\right)^{2}} \\
& =\left(n_{1} n_{2}+n_{1}-n_{2}-1\right) \sqrt{\left(n-n_{1}+1\right)^{2}+\left(n-n_{2}-1\right)^{2}}-n_{1} n_{2} \sqrt{\left(n-n_{1}\right)^{2}+\left(n-n_{2}\right)^{2}} \\
& +n_{1} \sum_{i=3}^{k} n_{i}\left[\sqrt{\left(n-n_{1}+1\right)^{2}+\left(n-n_{i}\right)^{2}}-\sqrt{\left(n-n_{1}\right)^{2}+\left(n-n_{i}\right)^{2}}\right] \\
& -n_{2} \sum_{i=3}^{k} n_{i}\left[\sqrt{\left(n-n_{2}\right)^{2}+\left(n-n_{i}\right)^{2}}-\sqrt{\left(n-n_{2}-1\right)^{2}+\left(n-n_{i}\right)^{2}}\right] \\
& +\sum_{i=3}^{k} n_{i}\left[\sqrt{\left(n-n_{2}-1\right)^{2}+\left(n-n_{i}\right)^{2}}-\sqrt{\left(n-n_{1}+1\right)^{2}+\left(n-n_{i}\right)^{2}}\right] .
\end{aligned}
$$

\section{Claim 3.}

$$
\left(n_{1} n_{2}+n_{1}-n_{2}-1\right) \sqrt{\left(n-n_{1}+1\right)^{2}+\left(n-n_{2}-1\right)^{2}}>n_{1} n_{2} \sqrt{\left(n-n_{1}\right)^{2}+\left(n-n_{2}\right)^{2}} .
$$

Proof of Claim 3. Since $n_{1} \geq n_{2}+2$, we have

$$
\left(n-n_{2}-1\right)^{2} \geq\left(n_{1}-1\right)^{2} \geq\left(n_{1}-1\right)\left(n_{2}+1\right)=n_{1} n_{2}+n_{1}-n_{2}-1>n_{1} n_{2},
$$

that is,

$$
\left(n-n_{1}+1\right)^{2}+\left(n-n_{2}-1\right)^{2}>n_{1} n_{2}
$$

that is,

$$
\frac{2\left(n_{1}-n_{2}-1\right)}{n_{1} n_{2}}>\frac{2\left(n_{1}-n_{2}-1\right)}{\left(n-n_{1}+1\right)^{2}+\left(n-n_{2}-1\right)^{2}}
$$

that is,

$$
1+\frac{2\left(n_{1}-n_{2}-1\right)}{n_{1} n_{2}}>\frac{\left(n-n_{1}\right)^{2}+\left(n-n_{2}\right)^{2}}{\left(n-n_{1}+1\right)^{2}+\left(n-n_{2}-1\right)^{2}}
$$

that is,

$$
\left(1+\frac{n_{1}-n_{2}-1}{n_{1} n_{2}}\right)^{2}>\frac{\left(n-n_{1}\right)^{2}+\left(n-n_{2}\right)^{2}}{\left(n-n_{1}+1\right)^{2}+\left(n-n_{2}-1\right)^{2}},
$$


which finishes the proof of Claim 3.

Claim 4. For $3 \leq i \leq k$,

$$
\begin{aligned}
& n_{1}\left[\sqrt{\left(n-n_{1}+1\right)^{2}+\left(n-n_{i}\right)^{2}}-\sqrt{\left(n-n_{1}\right)^{2}+\left(n-n_{i}\right)^{2}}\right] \\
& \quad>n_{2}\left[\sqrt{\left(n-n_{2}\right)^{2}+\left(n-n_{i}\right)^{2}}-\sqrt{\left(n-n_{2}-1\right)^{2}+\left(n-n_{i}\right)^{2}}\right] .
\end{aligned}
$$

Proof of Claim 4. Since $n_{1}-n_{2} \geq 2$, we have $2\left(n_{1}-n_{2}\right)\left(n-n_{1}-n_{2}\right)+n_{1}+n_{2}>0$, that is, $n_{1}\left[2\left(n-n_{1}\right)+1\right]>n_{2}\left[2\left(n-n_{2}\right)-1\right]$.

Moreover,

$$
\sqrt{\left(n-n_{1}+1\right)^{2}+\left(n-n_{i}\right)^{2}} \leq \sqrt{\left(n-n_{2}-1\right)^{2}+\left(n-n_{i}\right)^{2}}
$$

and

$$
\sqrt{\left(n-n_{1}\right)^{2}+\left(n-n_{i}\right)^{2}}<\sqrt{\left(n-n_{2}\right)^{2}+\left(n-n_{i}\right)^{2}}
$$

that is,

$$
\begin{aligned}
\sqrt{\left(n-n_{1}+1\right)^{2}+\left(n-n_{i}\right)^{2}}+\sqrt{\left(n-n_{1}\right)^{2}+\left(n-n_{i}\right)^{2}} \\
\quad<\sqrt{\left(n-n_{2}-1\right)^{2}+\left(n-n_{i}\right)^{2}}+\sqrt{\left(n-n_{2}\right)^{2}+\left(n-n_{i}\right)^{2}} .
\end{aligned}
$$

From the above results, we obtain

$$
\begin{gathered}
\frac{n_{1}\left[2\left(n-n_{1}\right)+1\right]}{\sqrt{\left(n-n_{1}+1\right)^{2}+\left(n-n_{i}\right)^{2}}+\sqrt{\left(n-n_{1}\right)^{2}+\left(n-n_{i}\right)^{2}}} \\
>\frac{n_{2}\left[2\left(n-n_{2}\right)-1\right]}{\sqrt{\left(n-n_{2}\right)^{2}+\left(n-n_{i}\right)^{2}}+\sqrt{\left(n-n_{2}-1\right)^{2}+\left(n-n_{i}\right)^{2}}},
\end{gathered}
$$

that is,

$$
\begin{aligned}
& \frac{n_{1}\left[\left(n-n_{1}+1\right)^{2}+\left(n-n_{i}\right)^{2}-\left(n-n_{1}\right)^{2}-\left(n-n_{i}\right)^{2}\right]}{\sqrt{\left(n-n_{1}+1\right)^{2}+\left(n-n_{i}\right)^{2}}+\sqrt{\left(n-n_{1}\right)^{2}+\left(n-n_{i}\right)^{2}}} \\
& \quad>\frac{n_{2}\left[\left(n-n_{2}\right)^{2}+\left(n-n_{i}\right)^{2}-\left(n-n_{2}-1\right)^{2}-\left(n-n_{i}\right)^{2}\right]}{\sqrt{\left(n-n_{2}\right)^{2}+\left(n-n_{i}\right)^{2}}+\sqrt{\left(n-n_{2}-1\right)^{2}+\left(n-n_{i}\right)^{2}}},
\end{aligned}
$$

which finishes the proof of Claim 4.

Since $n_{1} \geq n_{2}+2$, we have $n-n_{2}-1 \geq n-n_{1}+1$ and hence

$$
\sqrt{\left(n-n_{2}-1\right)^{2}+\left(n-n_{i}\right)^{2}} \geq \sqrt{\left(n-n_{1}+1\right)^{2}+\left(n-n_{i}\right)^{2}}
$$

for $3 \leq i \leq k$. Using the above result with Claims 3 and 4 , from (6), we obtain

$$
S O\left(K_{n_{1}-1, n_{2}+1, n_{3}, \ldots, n_{k}}\right)>S O\left(K_{n_{1}, n_{2}, n_{3}, \ldots, n_{k}}\right) .
$$

This completes the proof of the lemma.

We are now ready to proof of Theorem 2. 
Proof of Theorem 2. From the definition of chromatic number, any graph $G$ from $\mathcal{X}(n, k)$ has $k$ color classes each of which is an independent set. Suppose that these $k$ classes have order $n_{1}, n_{2}, \ldots, n_{k}$, respectively. By Lemma 1 , we obtain $S O(G) \leq S O\left(K_{n_{1}, n_{2}, \ldots, n_{k}}\right)$ with equality holding if and only if $G \cong K_{n_{1}, n_{2}, \ldots, n_{k}}$. We now apply Lemma 5 several times (if needed) and we obtain $S O\left(K_{n_{1}}, n_{2}, \ldots, n_{k}\right) \leq S O\left(T_{n}(k)\right)$ with equality holding if and only if $G \cong T_{n}(k)$. From the above two results with

$$
\begin{aligned}
S O\left(T_{n}(k)\right)= & r(k-r)\left\lfloor\frac{n}{k}\right\rfloor\left\lceil\frac{n}{k}\right\rceil \sqrt{\left(n-\left\lfloor\frac{n}{k}\right\rfloor\right)^{2}+\left(n-\left\lceil\frac{n}{k}\right\rceil\right)^{2}}+\sqrt{2}\left(\begin{array}{c}
r \\
2
\end{array}\right)\left\lceil\frac{n}{k}\right\rceil^{2}\left(n-\left\lceil\frac{n}{k}\right\rceil\right) \\
& +\sqrt{2}\left(\begin{array}{c}
k-r \\
2
\end{array}\right)\left\lfloor\frac{n}{k}\right\rfloor^{2}\left(n-\left\lfloor\frac{n}{k}\right\rfloor\right),
\end{aligned}
$$

we obtain the required result. This finishes the proof of this theorem.

Proof of Theorem 3. Let $S=\left\{v_{n-p+1}, v_{n-p+2}, \ldots, v_{n}\right\}(|S|=p)$ be the set of pendant vertices in $G$. Let $H_{n, n_{1}, \ldots, n_{n-p}}$ be a graph obtained from $G$ such that any two vertices $v_{i}$ and $v_{j}\left(v_{i}, v_{j} \in V(G) \backslash S, v_{i} v_{j} \notin E(G)\right)$ join by an edge, where $n_{i}(\geq 0,1 \leq i \leq n-p)$ is the number of pendant vertices adjacent to the vertex $v_{i}$ and $n_{1} \geq n_{2} \geq \cdots \geq n_{n-p}, n_{1}+n_{2}+$ $\cdots+n_{n-p}=p$. Then by Lemma 1 , one can easily see that $S O(G) \leq S O(H_{n,} \underbrace{n_{1}, \ldots, n_{n-p}})$. If $H_{n, n_{1}, \ldots, n_{n-p}} \cong K i_{n}^{n-p}$, then the equality holds in (2). Otherwise, $H_{n, n_{1}, \ldots, n_{n-p}}^{n_{1}} ¥$ $K_{i n}^{n-p}$. Let $H_{n,} \underbrace{n_{1}+1, n_{2}-1, \ldots, n_{n-p}} \cong H_{1}, H_{n,} \underbrace{n_{1}, n_{2}, \ldots, n_{n-p}} \cong H_{2}$. Since $n_{1} \geq n_{2}$, we obtain

$$
\begin{aligned}
& \mathrm{SO}\left(H_{1}\right)-\mathrm{SO}\left(\mathrm{H}_{2}\right) \\
& =\sum_{\substack{v_{k}: v_{1} v_{v} \in E\left(H_{1}\right) \\
d_{H_{1}}\left(v_{k}\right)=1}} \sqrt{d_{H_{1}}\left(v_{1}\right)^{2}+1}+\sum_{\substack{v_{k}: v_{1} v_{v} \in E\left(H_{1}\right) \\
d_{H_{1}}\left(v_{k}\right)>1}} \sqrt{d_{H_{1}}\left(v_{1}\right)^{2}+d_{H_{1}}\left(v_{k}\right)^{2}} \\
& +\sum_{\substack{v_{k}: v_{2} v_{v} \in E\left(H_{1}\right) \\
d_{H_{1}}\left(v_{k}\right)=1}} \sqrt{d_{H_{1}}\left(v_{2}\right)^{2}+1}+\sum_{\substack{v_{k}: v_{2} v_{v} \in E\left(H_{1}\right) \\
d_{H_{1}}\left(v_{k}\right)>1}} \sqrt{d_{H_{1}}\left(v_{1}\right)^{2}+d_{H_{1}}\left(v_{k}\right)^{2}} \\
& -\sum_{\substack{v_{k}: v_{1} v_{v} \in E\left(H_{2}\right) \\
d_{H_{2}}\left(v_{k}\right)=1}} \sqrt{d_{H_{2}}\left(v_{1}\right)^{2}+1}-\sum_{\substack{v_{k^{\prime}: v_{1}} v_{k} \in E\left(H_{2}\right) \\
d_{H_{2}}\left(v_{k}\right)>1}} \sqrt{d_{H_{2}}\left(v_{1}\right)^{2}+d_{H_{2}}\left(v_{k}\right)^{2}} \\
& -\sum_{\substack{v_{k}: v_{2} v_{v} \in E\left(H_{2}\right) \\
d_{H_{2}}\left(v_{k}\right)=1}} \sqrt{d_{H_{2}}\left(v_{2}\right)^{2}+1}-\sum_{\substack{v_{k}: v_{2} v_{v} \in E\left(H_{2}\right) \\
d_{H_{2}}\left(v_{k}\right)>1}} \sqrt{d_{H_{2}}\left(v_{2}\right)^{2}+d_{H_{2}}\left(v_{k}\right)^{2}} \\
& =\left(n_{1}+1\right) \sqrt{\left(n+n_{1}-p\right)^{2}+1}+\sum_{i=3}^{n-p} \sqrt{\left(n+n_{1}-p\right)^{2}+\left(n+n_{i}-p-1\right)^{2}} \\
& +\left(n_{2}-1\right) \sqrt{\left(n+n_{2}-p-2\right)^{2}+1}+\sum_{i=3}^{n-p} \sqrt{\left(n+n_{2}-p-2\right)^{2}+\left(n+n_{i}-p-1\right)^{2}} \\
& -n_{1} \sqrt{\left(n+n_{1}-p-1\right)^{2}+1}-\sum_{i=3}^{n-p} \sqrt{\left(n+n_{1}-p-1\right)^{2}+\left(n+n_{i}-p-1\right)^{2}} \\
& -n_{2} \sqrt{\left(n+n_{2}-p-1\right)^{2}+1}-\sum_{i=3}^{n-p} \sqrt{\left(n+n_{2}-p-1\right)^{2}+\left(n+n_{i}-p-1\right)^{2}} \\
& +\sqrt{\left(n+n_{1}-p\right)^{2}+\left(n+n_{2}-p-2\right)^{2}}-\sqrt{\left(n+n_{1}-p-1\right)^{2}+\left(n+n_{2}-p-1\right)^{2}} .
\end{aligned}
$$

Claim 5. $n_{1} \sqrt{a^{2}+1}+n_{2} \sqrt{(b-1)^{2}+1}>n_{1} \sqrt{(a-1)^{2}+1}+n_{2} \sqrt{b^{2}+1}$, where $a=$ $n+n_{1}-p$ and $b=n+n_{2}-p-1$. 
Proof of Claim 5. First we assume that $n_{1}=n_{2}$. Thus, we have $a=b+1$. In this case we have to prove that

$$
\sqrt{(b+1)^{2}+1}+\sqrt{(b-1)^{2}+1}>\sqrt{b^{2}+1}+\sqrt{b^{2}+1},
$$

that is,

$$
\sqrt{(b+1)^{2}+1}-\sqrt{b^{2}+1}>\sqrt{b^{2}+1}-\sqrt{(b-1)^{2}+1},
$$

that is,

$$
2 b+\sqrt{b^{2}+1} \sqrt{(b-1)^{2}+1}>\sqrt{b^{2}+1} \sqrt{(b+1)^{2}+1}
$$

that is,

$$
\sqrt{b^{2}+1} \sqrt{(b-1)^{2}+1}>b^{2}-b+1,
$$

after squaring both sides, one can easily check that the above result is true. Hence the Claim 5 is true for $n_{1}=n_{2}$.

Next we assume that $n_{1} \geq n_{2}+1$. In this case we have to prove that

$$
n_{1}\left(\sqrt{a^{2}+1}-\sqrt{(a-1)^{2}+1}\right)>n_{2}\left(\sqrt{b^{2}+1}-\sqrt{(b-1)^{2}+1}\right) .
$$

One can easily see that

$$
\sqrt{b^{2}+1}-\sqrt{(b-1)^{2}+1}<1 .
$$

Using this, from the above, we have to prove that

$$
\frac{n_{1}}{n_{2}}>\frac{1}{\sqrt{a^{2}+1}-\sqrt{(a-1)^{2}+1}}
$$

that is,

$$
\sqrt{a^{2}+1}>\sqrt{(a-1)^{2}+1}+\frac{n_{2}}{n_{2}+1}
$$

that is,

$$
2 a-1>\frac{2 n_{2}}{n_{2}+1} \sqrt{(a-1)^{2}+1}+\left(\frac{n_{2}}{n_{2}+1}\right)^{2},
$$

that is,

$$
2 a-2>\frac{2 n_{2}}{n_{2}+1} \sqrt{(a-1)^{2}+1}
$$

that is,

$$
(a-1)^{2}\left(n_{2}+1\right)^{2}>n_{2}^{2}\left((a-1)^{2}+1\right)
$$

that is,

$$
(a-1)^{2}\left(2 n_{2}+1\right)>n_{2}^{2},
$$

which is always true as $a \geq n_{1}+1 \geq n_{2}+2$. This completes the proof of Claim 5 .

Claim 6. $\sqrt{(r+1)^{2}+t^{2}}+\sqrt{(s-1)^{2}+t^{2}}>\sqrt{r^{2}+t^{2}}+\sqrt{s^{2}+t^{2}}$, where $r=n+n_{1}-p-1$, $s=n+n_{2}-p-1$ and $t=n+n_{i}-p-1$.

Proof of Claim 6. We have to prove that

$$
\sqrt{(r+1)^{2}+t^{2}}-\sqrt{s^{2}+t^{2}}>\sqrt{r^{2}+t^{2}}-\sqrt{(s-1)^{2}+t^{2}},
$$


that is,

$$
\begin{aligned}
& (r+1)^{2}+t^{2}+s^{2}+t^{2}-2 \sqrt{(r+1)^{2}+t^{2}} \sqrt{s^{2}+t^{2}} \\
& >r^{2}+t^{2}+(s-1)^{2}+t^{2}-2 \sqrt{r^{2}+t^{2}} \sqrt{(s-1)^{2}+t^{2}}
\end{aligned}
$$

that is,

$$
\left(r+s+\sqrt{r^{2}+t^{2}} \sqrt{(s-1)^{2}+t^{2}}\right)^{2}>\left((r+1)^{2}+t^{2}\right)\left(s^{2}+t^{2}\right),
$$

that is,

$$
\sqrt{r^{2}+t^{2}} \sqrt{(s-1)^{2}+t^{2}}>r(s-1)+t^{2},
$$

that is,

$$
(r-s+1)^{2}>0,
$$

which is true always. This completes the proof of Claim 6.

Claim 7. $\sqrt{\left(n+n_{1}-p\right)^{2}+\left(n+n_{2}-p-2\right)^{2}}>\sqrt{\left(n+n_{1}-p-1\right)^{2}+\left(n+n_{2}-p-1\right)^{2}}$.

Proof of Claim 7. We have to prove that

$$
\left(n+n_{1}-p\right)^{2}+\left(n+n_{2}-p-2\right)^{2}>\left(n+n_{1}-p-1\right)^{2}+\left(n+n_{2}-p-1\right)^{2},
$$

that is,

$$
2\left(n+n_{1}-p\right)>2\left(n+n_{2}-p\right)-2
$$

that is,

$$
n_{1}-n_{2}+1>0,
$$

which is true always. This completes the proof of Claim 7.

By Claim 5, we obtain

$$
\begin{aligned}
& \left(n_{1}+1\right) \sqrt{a^{2}+1}+\left(n_{2}-1\right) \sqrt{(b-1)^{2}+1}-n_{1} \sqrt{(a-1)^{2}+1}-n_{2} \sqrt{b^{2}+1} \\
& =n_{1} \sqrt{a^{2}+1}+n_{2} \sqrt{(b-1)^{2}+1}-n_{1} \sqrt{(a-1)^{2}+1}-n_{2} \sqrt{b^{2}+1} \\
& \quad+\sqrt{a^{2}+1}-\sqrt{(b-1)^{2}+1} \\
& >\sqrt{a^{2}+1}-\sqrt{(b-1)^{2}+1}>0 .
\end{aligned}
$$

By Claim 6, we obtain

$$
\begin{aligned}
& \sum_{i=3}^{n-p}\left[\sqrt{\left(n+n_{1}-p\right)^{2}+\left(n+n_{i}-p-1\right)^{2}}+\sqrt{\left(n+n_{2}-p-2\right)^{2}+\left(n+n_{i}-p-1\right)^{2}}\right. \\
& \left.-\sqrt{\left(n+n_{1}-p-1\right)^{2}+\left(n+n_{i}-p-1\right)^{2}}-\sqrt{\left(n+n_{2}-p-1\right)^{2}+\left(n+n_{i}-p-1\right)^{2}}\right] \\
& >0 .
\end{aligned}
$$

Using (8), (9) with Claim 7 in (7), we obtain $S O\left(H_{1}\right)>S O\left(H_{2}\right)$. Using this result several times (if needed), we obtain

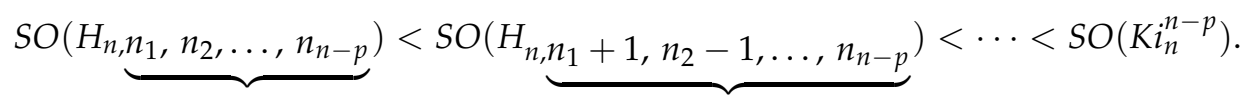

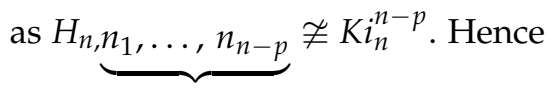

$$
S O(G) \leq S O(H_{n,} \underbrace{n_{1}, \ldots, n_{n-p}})<S O\left(K i_{n}^{n-p}\right)
$$


This completes the proof of the theorem.

\section{Conclusions}

Sombor index was used to model entropy and enthalpy of vaporization of alkanes with satisfactory prediction potential, indicating that this topological index may be used successfully on modeling thermodynamic properties of compounds. In this paper we presented some lower and upper bounds on the Sombor index of graph $G$ in terms of graph parameters (clique number, chromatic number, number of pendant vertices, etc.) and characterize the extremal graphs. Here we pose two related problems.

Problem 1. Characterize the maximal graph with respect to Sombor index among all connected graphs of order $n$ with clique number $\omega$.

Problem 2. Characterize the minimal graph with respect to Sombor index among all connected graphs of order $n$ with $p$ pendant vertices.

Author Contributions: Conceptualization, K.C.D. and Y.S.; investigation, K.C.D. and Y.S.; writingoriginal draft preparation, K.C.D. and Y.S.; writing — review and editing, K.C.D. and Y.S. All authors have read and agreed to the published version of the manuscript.

Funding: This research received no external funding.

Institutional Review Board Statement: Not applicable.

Informed Consent Statement: Not applicable.

Data Availability Statement: Not applicable.

Conflicts of Interest: The authors declare no conflict of interest.

\section{References}

1. Bondy, J.A.; Murty, U.S.R. Graph Theory with Applications; MacMillan: New York, NY, USA, 1976.

2. Trinajsti c, N. Chemical Graph Theory; CRC Press: Boca Raton, FL, USA, 1983; Volume I/II.

3. Todeschini, R.; Consonni, V. Handbook of Molecular Descriptors; Wiley-VCH: Weinheim, Germany, 2000.

4. Borovićanin, B.; Das, K.C.; Furtula, B.; Gutman, I. Zagreb indices: Bounds and extremal graphs. MATCH Commun. Math. Comput. Chem. 2017, 78, 17-100.

5. Buyantogtokh, L.; Horoldagva, B.; Das, K.C. On reduced second Zagreb index. J. Combin. Opt. 2020, 39, 776-791. [CrossRef]

6. Das, K.C. Maximizing the sum of the squares of the degrees of a graph. Discrete Math. 2004, 285, 57-66. [CrossRef]

7. Das, K.C. On comparing Zagreb indices of graphs. MATCH Commun. Math. Comput. Chem. 2010, 63, 433-440.

8. Das, K.C.; Ali, A. On a conjecture about the second Zagreb index. Discret. Math. Lett. 2019, 2, 38-43.

9. Das, K.C.; Gutman, I. Some properties of the Second Zagreb Index. MATCH Commun. Math. Comput. Chem. 2004, 52, $103-112$.

10. Das, K.C.; Gutman, I. On Sombor Index of Trees, Submitted. Available online: https:/ /www.researchgate.net/publication/3517 01470_On_Sombor_index_of_trees (accessed on 24 May 2021).

11. Das, K.C.; Gutman, I.; Horoldagva, B. Comparison between Zagreb indices and Zagreb coindices. MATCH Commun. Math. Comput. Chem. 2012, 68, 189-198.

12. Das, K.C.; Gutman, I.; Zhou, B. New upper bounds on Zagreb indices. J. Math. Chem. 2009, 46, 514-521. [CrossRef]

13. Das, K.C.; Xu, K.; Gutman, I. On Zagreb and Harary indices. MATCH Commun. Math. Comput. Chem. 2013, 70, 301-314.

14. Deng, H.; Tang, Z.; Wu, R. Molecular trees with extremal values of Sombor indices. Int. J. Quantum Chem. 2021, 11, e26622.

15. Shang, Y. Laplacian Estrada and normalized Laplacian Estrada indices of evolving graphs. PLoS ONE 2015, 10, e0123426. [CrossRef] [PubMed]

16. Xu, K.; Gao, F.; Das, K. C.; Trinajstić, N. A formula with its applications on the difference of Zagreb indices of graphs. J. Math. Chem. 2019, 57, 1618-1626. [CrossRef]

17. Xu, K.; Das, K. C.; Balachandran, S. Maximizing the Zagreb indices of $(n, m)$-graphs. MATCH Commun. Math. Comput. Chem. 2014, 72, 641-654.

18. Gutman, I. Geometric approach to degree-based topological indices: Sombor indices. MATCH Commun. Math. Comput. Chem. 2021, 86, 11-16.

19. Cruz, R.; Gutman, I.; Rada, J. Sombor index of chemical graphs, Appl. Math. Comput. 2021, 339, 126018.

20. Cruz, R.; Rada, J. Extremal values of the Sombor index in unicyclic and bicyclic graphs. J. Math. Chem. 2021, 59, 1098-1116. [CrossRef]

21. Das, K.C.; Çevik, A.S.; Cangul, I.N.; Shang, Y. On Sombor index. Symmetry 2021, 13, 140. [CrossRef]

22. Gutman, I. Some basic properties of Sombor indices. Open J. Discret. Appl. Math. 2021, 4, 1-3. [CrossRef] 
23. Milovanović, I.; Milovanović, E.; Matejić, M. On some mathematical properties of Sombor indices. Bull. Int. Math. Virtual Inst. 2021, 11, 341-353.

24. Redžepović, I. Chemical applicability of Sombor indices. J. Serbian Chem. Soc. 2021. [CrossRef]

25. Réti, T.; Došlić, T.; Ali, A. On the Sombor index of graphs. Contrib. Math. 2021, 3, 11-18.

26. Wang, Z.; Mao, Y.; Li, Y.; Furtula, B. On relations between Sombor and other degree-based indices. J. Appl. Math. Comput. 2021. [CrossRef] 